Ausbildung

\title{
Welche Ethikkompetenzen braucht die Pflege?
}

— Ethische Fragestellungen und Konflikte gehören in allen pflegeberuflichen Handlungsfeldern zum Alltag. Zukünftige Pflegende und Studierende der Pflege sollen darauf vorbereitet sein. Die entsprechenden Handlungskompetenzen dafür müssen in Ausbildung und Studium vermittelt werden. Doch was sind die zentralen Ethikkompetenzen pflegeberuflichen Handelns? Welche Ethikkompetenzen braucht es in der Pflege, um professionell - das heißt fachlich fundiert und ethisch reflektiert - zu entscheiden und zu handeln? Wie lässt sich ein Ausbildungsziel erreichen, das im aktuell diskutierten Pflegeberufereformgesetz ausdrücklich formuliert wird? Danach soll die Pflege "entsprechend dem allgemein anerkannten Stand pflegewissenschaftli- cher, medizinischer und weiterer bezugswissenschaftlicher Erkenntnisse auf der Grundlage einer professionellen Ethik" erfolgen.

Mit einer Konkretisierung der Ethikkompetenzen in der Pflege hat sich nun die Sektion Lehrende im Bereich der Pflegeausbildung und der Pflegestudiengänge in der Akademie für Ethik in der Medizin e.V. im Auftrag des Vorstandes der AEM befasst. Zentrale Aspekte der Ethikkompetenz wurden konkret formuliert und die wesentlichen Konturen einer professionellen Ethik dargelegt. Das Papier der Sektion konkretisiert die Bedeutung und den Gegenstand der pflegeethischen Kompetenzen. Abschließend sind sechs zentrale Bildungsziele formuliert - zum Beispiel die
Sensibilität für ethische Konfliktsituationen im pflegeberuflichen Handeln, aber auch die Empathiefähigkeit und die Fähigkeit zum interdisziplinären Perspektivenwechsel. Ziel des Papiers ist es, eine Orientierung für die Ausgestaltung und Entwicklung von Curricula und zukünftigen (Rahmen-)Lehrplänen zu bieten. Das Papier ermöglicht es, die geforderten Ethikkompetenzen zu erfassen und für die jeweilige Qualifizierungsmaßnahmen entsprechend zu konkretisieren.

Die Empfehlungen der Sektion sind unter dem Titel "Zentrale Aspekte der Ethikkompetenz in der Pflege" seit Oktober 2016 in der Zeitschrift Ethik in der Medizin publiziert. Sie können bis Ende Januar kostenlos auf SpringerLink heruntergeladen werden (http://link.springer.com/article/10.1007/ s00481-016-0415-7).

www.aem-online.de

\section{Gehaltserhöhung \\ $3.000 €$ für jede Pflegefachkraft}

— Die Münchenstift GmbH zahlt ab 01. Januar 2017 allen Pflegefachkräften ab dem ersten Tag $3.000 €$. Nach zwei Jahren Verhandlungen und intensiven Restrukturierungsmaßnahmen konnten sich die Münchenstift und ver.di auf dieses bundesweit einmalige Tarifgesamtwerk einigen. „Pflegekräfte, die mit Herz und Engagement bei uns arbeiten, müssen sich München auch leisten können. Alle fordern die bessere Bezahlung in der Pflege - wir tun es", erklärt der Geschäftsführer Siegfried Benker. Laut Benker soll diese übertarifliche Bezahlung auch dazu beitragen, dass mehr von den durchschnittlich 250 Azubis nach der Ausbuildung im Unternehmen gehalten werden. Die Münchenstift $\mathrm{GmbH}$ ist mit rund 1.800 Mitarbeitern eines der größten Dienstleistungsunternehmen für Senioren in München.

www.muenchenstift.de

\section{Bühnenarbeit}

\section{Schüler, Theater und Demenz}

— Bereits zum zweiten Mal fand im Oktober am kooperativen Bildungszentrum für Gesundheitsberufe am Carl-von-BasedowKlinikum in Merseburg ein Theaterprojekt zum Thema Demenz für und mit Auszubildenden statt. Gemeinsam mit der Berliner Theaterregisseurin Martha Hölters-Freier entwickelten die Schüler im zweiten Ausbildungsjahr der Gesundheits- und Krankenpflege ein Stück, das auf eigene Erfahrungen aufbaut, literarische Bruchstücke einbezieht und einen Perspektivwechsel initiiert. Die Erarbeitung von Szenen, Bildern und Bewegungssequenzen zum Leben mit Demenz sollte einen Lernraum öffnen, in dem sich die Schüler auch irrational-emotional und körperlich mit ihren Pflegeerfahrungen auseinandersetzen konnten. Das Verständnis der Darsteller für die Befindlichkeiten ihrer Pflegebefohlenen ist dabei gewachsen, eine neue menschliche Nähe zu ihnen hat sich hergestellt. Begeistert zeigt sich Schulleiterin Ines Schiller, die diese Unterrichtsmethode in den Lehrplan integriert und das Projekt begleitet hat. „Unsere Auszubildenden haben in der Praxis oft Kontakt zu Menschen mit Verwirrtheitszuständen unterschiedlicher Ursachen. Unser Ziel war es, Verständnis für sie, ihre Gefühlswelt und das Zeitlupentempo zu vermitteln. Das ist offensichtlich gelungen." Herausgekommen ist ein Stück mit dem doppelsinnigen Titel: „ES NEBELT - Bilder und Szenen aus dem Leben mit Demenz".

www.klinikum-saalekreis.de

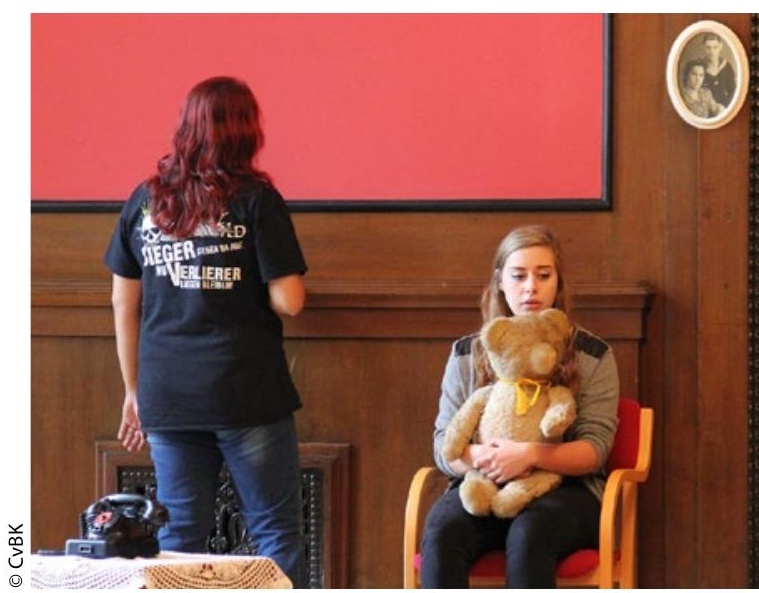

Theaterarbeit als Mittel der Empathiefähigkeitentwicklung als besondere Lernmethode. Die 16 Auszubildenden erlangten während der Projektwoche ein größeres Verständnis für die Gefühlswelt der Demenzkranken. 